\title{
Phase scintillation observation during coronal sounding experiments with NOZOMI spacecraft
}

\author{
T. Imamura ${ }^{1}$, K. Noguchi ${ }^{2}$, A. Nabatov ${ }^{3, \star}$, K.-I. Oyama ${ }^{1}$, Z. Yamamoto ${ }^{1}$, and M. Tokumaru ${ }^{4}$ \\ ${ }^{1}$ Institute of Space and Astronautical Science, Japan Aerospace Exploration Agency, 3-1-1, Yoshinodai, Sagamihara, \\ Kanagawa 229-8510, Japan \\ e-mail: ima@isas.jaxa.jp \\ 2 Nara Women's University, Kita-uoya Nishi-machi, Nara 630-8506, Japan \\ 3 Institute of Radio Engineering and Electronics of the Russian Academy of Sciences, Russia \\ ${ }^{4}$ Solar-Terrestrial Environment Laboratory, Nagoya University, Honohara 3-13, Toyokawa, Aichi 442-8507, Japan
}

Received 26 December 2004 / Accepted 11 April 2005

\begin{abstract}
Radio occultation observations of the solar corona at solar offset distances of 12.8-36.9 $R_{\mathrm{S}}$ were performed from December of 2000 to January of 2001, using the Nozomi spacecraft (Planet-B) of Japan. Phase scintillation spectra up to the frequency of $\sim 10 \mathrm{~Hz}$ were obtained in two-way mode with $S$-band uplink and $X$-band downlink. The spectra cannot be represented by a single power law especially for small offset distances. That is, at low frequencies (large scales) the spectra show slopes indicative of Kolmogorov, a local flattening occurs from $\sim 0.5 \mathrm{~Hz}$ (scale $\sim 600 \mathrm{~km})$ to $\sim 3 \mathrm{~Hz}(\sim 100 \mathrm{~km})$, and a steepening occurs again at higher frequencies. This three-component nature of the spectrum was observed with a single method for the first time, although the spectral shape is variable and the three-component feature is not always evident.
\end{abstract}

Key words. Sun: corona - Sun: solar wind - turbulence

\section{Introduction}

Information on the small-scale structures of plasma near the sun is important for understanding the mechanism of heating the plasma and accelerating the solar wind. Observations of spacecraft radio signals during solar conjunctions can provide great deal of information on such structures which cannot be investigated directly by spacecraft (Coles \& Harmon 1989; Yakovlev 2002). One of the most valuable pieces of information provided by those measurements is the phase scintillation, in which the phase, which is proportional to the change in the electron content along the ray path, can be spectral analyzed to investigate the spectral shape of the electron density fluctuations in the solar corona. If the three-dimensional density spectrum is represented by a power law with spectral index $p$, the phase fluctuation spectrum also follows a power law with the slope $1-p$ (Woo et al. 1976a).

Woo \& Armstrong (1979) have analyzed the Viking radio science data set with solar offset distances $2.17-215 R_{\mathrm{S}}$ $\left(R_{\mathrm{S}} \equiv\right.$ solar radii) and found that the Kolmogorov-like spectral slope $p=11 / 3$ prevails for distances $>20 R_{\mathrm{S}}$ and less slope prevails for smaller distances. By combining the results of various observation techniques that provide information on different scales, Coles \& Harmon (1989) and

^ Now at the Radio Astronomical Institute of the National Academy of Sciences of Ukraine, Ukraine.
Coles et al. (1991) proposed a three-component model of the density spectrum: a Kolmogorov-like spectrum in the scale range $10^{6}-10^{3} \mathrm{~km}$; a flattening at scales between $10^{3} \mathrm{~km}$ and the inner scale of $10-100 \mathrm{~km}$; and a steepened part at smaller scales. In the analysis of the Ulysses radio signals that sounded $25 R_{\mathrm{S}}$, Pätzold et al. (1996) suggested that the spectral slope depends on the solar latitude and that a local flattening occurs at frequencies $>0.06 \mathrm{~Hz}$ and $>0.2 \mathrm{~Hz}$ for the coronal hole and the streamer spectra, respectively. Their observation seems to be consistent with the suggestion by Coles \& Harmon (1989), although the steepened part at higher frequencies was beyond the Nyquist frequency of $0.5 \mathrm{~Hz}$. More studies are needed to constrain the spectral shape over wide scale ranges in various solar wind conditions.

In the present paper we report the results of phase scintillation observations of the solar corona at solar offset distances of 12.8-36.9 $R_{\mathrm{S}}$ using the two-way radio signals of the Japanese spacecraft "Nozomi" (Planet-B). The relatively wide spectral coverage of the phase measurement, which extends to $\sim 10 \mathrm{~Hz}$, allows us to observe the detailed structure of the phase spectrum.

\section{Observation}

The Nozomi spacecraft (Planet-B) was launched in 1998 and was planned to become a Mars orbiter. Unfortunately the 
Table 1. Observation conditions of the Nozomi radio propagation experiment. Runs in December of 2000 belong to the ingress path, and those in January of 2001 belong to the egress path. "Time" is the start time of recording signals, "Length" the DFT lengths in minutes for calculating phase spectra, and $R$ the solar offset distance. The coronal regions sounded were determined from the images taken by SOHO/LASCO (see text).

\begin{tabular}{ccccc}
\hline \hline Date & Time (UT) & Length $(\mathrm{min})$ & $R\left(R_{\mathrm{S}}\right)$ & Coronal region \\
\hline Dec. 06, 2000 & $00: 51$ & 56 & 36.9 & between streamers \\
Dec. 11, 2000 & $01: 03$ & 56 & 31.5 & CME \\
Dec. 16, 2000 & $01: 04$ & 56 & 26.0 & between streamers \\
Dec. 18, 2000 & $01: 08$ & 28 & 23.8 & between streamers \\
Dec. 23, 2000 & $00: 51$ & 56 & 18.3 & between streamers \\
Dec. 27, 2000 & $00: 52$ & 56 & 13.9 & between streamers \\
Dec. 28, 2000 & $00: 51$ & 56 & 12.8 & between streamers \\
Jan. 22, 2001 & $06: 18$ & 14 & 15.7 & small coronal holes \\
Jan. 23, 2001 & $05: 23$ & 28 & 16.8 & small coronal holes \\
\hline
\end{tabular}

injection of the spacecraft into the Mars orbit failed due to malfunction of the onboard electronics. However, radio science experiments were successfully performed when the line-of-sight to the spacecraft approached the sun (solar conjunction) during the period from December of 2000 to January of 2001. Table 1 summarizes the observation conditions, and Fig. 1 illustrates the geometry of the solar conjunction in the sky projection. The observation was stopped for 24 days around the closest approach to the sun, since the antenna pointing system of the spacecraft was not designed to maintain the radio link when the angle between the sun and the earth viewed from the spacecraft becomes small. The solar offset distance varied from 36.9 to $12.8 R_{\mathrm{S}}$ during the ingress observation, and from 15.7 to $16.8 R_{\mathrm{S}}$ during the egress observation.

The coronal regions sounded by the ray path (Table 1) were determined from the C3 coronagraph images of the Large Angle and Spectrometric Coronagraph (LASCO) onboard the SOHO spacecraft (Brueckner et al. 1995), which are distributed through the www page (http://sohowww.nascom.nasa.gov). According to the images, the ray paths in the ingress occultation were located between small streamers, while the ray paths in the egress passed through small coronal holes. The experiment at $31.5 R_{\mathrm{S}}$ (December 11) seems to be influenced by a coronal mass ejection (CME) which occurred in the northwest quadrant of the solar corona.

In contrast to the previous phase scintillation observations which used dual-frequency phase coherent systems (Woo \& Armstrong 1979; Coles et al. 1991; Pätzold et al. 1996), our experiments were performed in a two-way mode with $S$-band $(2.1123 \mathrm{GHz})$ uplink and $X$-band $(8.4109 \mathrm{GHz}, 880 / 221$ times the uplink frequency) downlink. Although the spacecraft was equipped with a coherent dual-frequency downlink system using an onboard ultra-stable oscillator (Oyama et al. 2001), the $S$-band transmitter and the ultra-stable oscillator were not available during the solar conjunction due to another malfunction of the onboard electronics. The uplink signal was generated using a hydrogen maser (Allan deviation $<3 \times 10^{-13}$ for $1 \mathrm{~s},<3 \times 10^{-15}$ for $10^{3} \mathrm{~s}$ ) at the Usuda Deep Space Center (UDSC) of Japan.

The signal received by the spacecraft was converted to $X$-band by an onboard transponder. The bandwidth of the phase-locked loop of the transponder was wider than $20 \mathrm{~Hz}$;

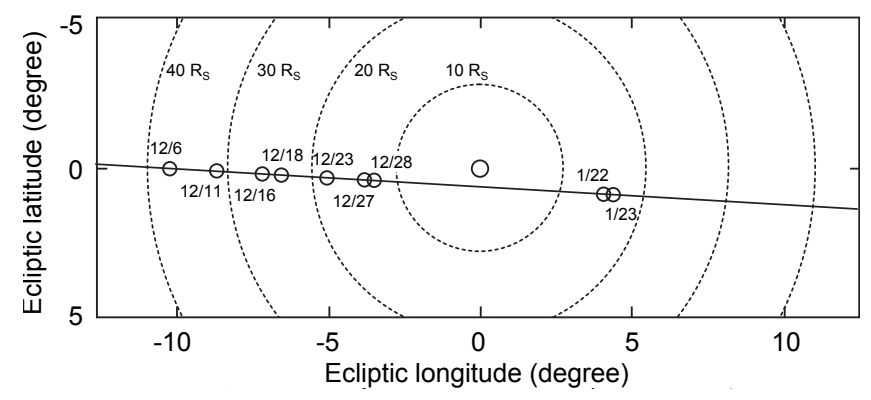

Fig. 1. Geometry of the Nozomi solar conjunction from December of 2000 to January of 2001. The solid line indicates the trajectory of the spacecraft with respect to the sun on the plane of the sky, the circles indicate the locations where observations were made, and dotted concentric circles are constant solar offset distance contours drawn every $10 R_{\mathrm{S}}$.

therefore, the phase fluctuations in the uplink signal should be reflected in those of the downlink signal at least up to this frequency. The reduction of the phase power due to the transponder's performance is smaller than $3 \mathrm{~dB}$ below $20 \mathrm{~Hz}$, and the distortion is negligible below $10 \mathrm{~Hz}$ except for a small hump with $<1-2 \mathrm{~dB}$ around $10 \mathrm{~Hz}$. The signal received at the UDSC was converted to $\sim 20 \mathrm{kHz}$ by an open-loop heterodyne system stabilized by the hydrogen maser and digitized with a sampling rate of $80 \mathrm{kHz}$. Since the $S$-band is 880/221 times more sensitive to plasma than the $X$-band and since the phase fluctuations in the uplink signal are amplified by a factor of $880 / 221$ by the transponder, the density fluctuations along the uplink path should have $(880 / 221)^{4}$ times greater influence on the observed phase power than those along the downlink path.

Figure 2 shows the development of the signal spectrum during the solar conjunction. The spectral broadening bandwidth increases as the distance to the sun decreases and attains a value $4.9 \mathrm{~Hz}$, which is $\sim 5$ times larger than the typical one-way $S$-band bandwidths for similar solar offset distances (Woo \& Armstrong 1979). This is consistent with the expectation that the observed frequency (phase) fluctuations should basically represent the disturbances on the uplink signal rather than those on the downlink signal. The relatively broad bandwidth at $31.5 R_{\mathrm{S}}$ (December 11) is attributed to the influence of the CME described before: the bandwidth increases with the rms fluctuation of the electron density and the solar wind speed 

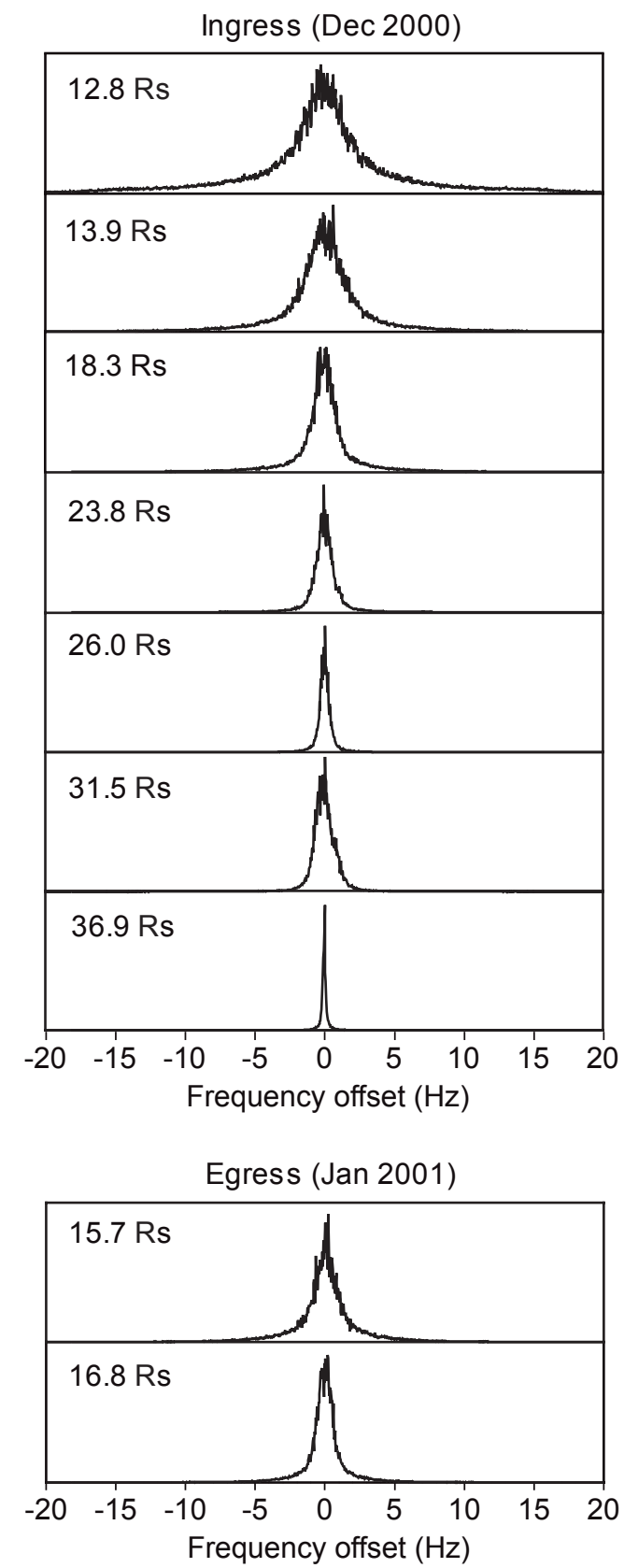

Fig. 2. Carrier spectra observed during the solar conjunction. Each spectrum is the result of $30 \mathrm{~min}$ of observation in two-way mode with $S$-band uplink and $X$-band downlink. Numerals in the panels indicate solar offset distances.

(Woo et al. 1976b), both of which will increase in CMEs. We do not analyze the detailed spectral shape of the received signal, which also has information on the density spectrum (Woo \& Armstrong 1979), since the performance of the transponder in converting the spectral shape is complicated and difficult to handle.

During the solar conjunction, interplanetary scintillation (IPS) measurements of the solar wind speed have also been made by the Nagoya university group together with our observation. Their results are utilized in the interpretation of the phase scintillation spectra.

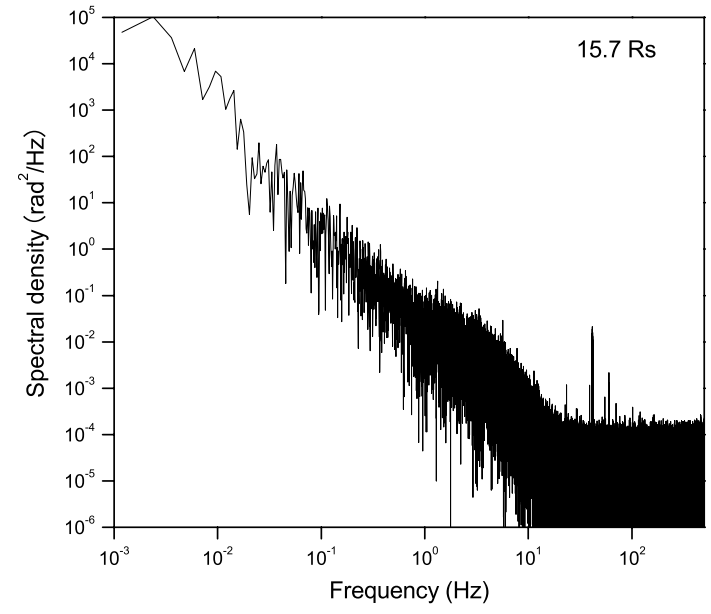

Fig. 3. Phase scintillation spectrum obtained on January 22, 2001, when the solar offset distance was $15.7 R_{\mathrm{S}}$. Spectral densities are for the $S$-band uplink path, converted from the observed $X$-band spectral densities by multiplying by a factor $(221 / 880)^{2}$.

\section{Phase scintillation spectra}

The phase scintillation spectrum for each observation run was obtained in the following manner. First the carrier frequencies of successive short time intervals are determined by fitting a theoretical signal spectrum to the discrete Fourier transform (DFT) of successive blocks of the voltage time series (Lipa \& Tyler 1979), thereby obtaining an approximate frequency time series. Next a third-order polynomial was fitted to the frequency time series above, and the frequency variation corresponding to the polynomial was subtracted from the original signal by heterodyning. Via this procedure we have subtracted the long-term frequency drift due to the orbital motions of the spacecraft and the earth and the large-scale variation of the electron content integrated along the ray path. In the course of the data reduction, the signal was bandpass filtered repeatedly to increase the signal-to-noise ratio, and at the same time decimated. This filtering scheme automatically converts the data to a complex representation (analytic signal) in which each datum comprises the amplitude and phase of the signal with noise. The low-noise analytic signal after filtering directly yields the phase by removing cycle slips via phase unwrapping procedure (Mizuno et al. 1992). The resultant phase time series, having a sampling interval of $0.0008 \mathrm{~s}$, was converted to a phase power spectrum using DFT including a Welch window.

An example of the calculated phase spectra is shown in Fig. 3, with spectral densities scaled to the $S$-band uplink by multiplying $(221 / 880)^{2}$. The magnitudes of the spectral densities are similar to those of previous results (Woo \& Armstrong 1979; Pätzold et al. 1996). In this figure the non-power-law spectral feature is evident, with a flattened part at $0.5-3 \mathrm{~Hz}$ and a steepened part at $>3 \mathrm{~Hz}$. The occurrence of steepening at $>3 \mathrm{~Hz}$ cannot be attributed to the performance of the transponder, since the distortion caused by the transponder is much less in this frequency region. At $>30 \mathrm{~Hz}$ the signal flattens into a white noise spectrum. We estimated the noise level by averaging the $200-500 \mathrm{~Hz}$ part and then subtracted it from the 
remaining part of the spectrum for all runs. Hereafter we show only the frequency region $<10 \mathrm{~Hz}$ where the influence of the transponder is negligible.

To examine the reliability of the analysis, the phase spectra obtained above were compared with those calculated from the frequency time series, each of which was obtained by successively fitting a theoretical spectrum again to the DFT of the successive blocks of the filtered low-noise data. This frequency time series, having a sampling interval of $0.0512 \mathrm{~s}$, was converted to a frequency power spectrum using DFT, and was further converted to a phase power spectrum by dividing it by square frequencies. It was found that the two methods give virtually the same result in the frequency region where they overlap (not shown here).

Figure 4 compares the phase scintillation spectra for all observing runs after smoothing the curves for legibility. The result for the solar offset distance of $114 R_{\mathrm{S}}(0.53 \mathrm{AU})$ taken on June 2, 2000 is also plotted as a kind of calibration spectrum: the sufficiently low level of this spectrum compared to those of others suggests that the spectra taken during the solar conjunction principally reflect the influence of near-sun plasma. The spatial wavelengths corresponding to the frequencies are also shown in Fig. 4 assuming a solar wind speed of $300 \mathrm{~km} \mathrm{~s}^{-1}$. According to the IPS observations made simultaneously using the Nozomi spacecraft by the Nagoya university group (Tokumaru et al., submitted), the radial wind speed ranged between 200 and $540 \mathrm{~km} \mathrm{~s}^{-1}$ and the majority are below $400 \mathrm{~km} \mathrm{~s}^{-1}$, which is typical of the solar maximum phase.

Figure 4 indicates that the scintillation level increases as the solar offset distance decreases, except the relatively high level at $31.5 R_{\mathrm{S}}$ (December 11), which will be attributed to the influence of a CME. The scintillation seems to be slightly weaker in coronal holes than in other regions, as the level at $16.8 R_{\mathrm{S}}$ in the egress (small coronal holes) is close to that at $18.3 R_{\mathrm{S}}$ in the ingress.

It is evident that the spectra cannot be represented by a single power law especially for the distances $<20 R_{\mathrm{S}}$. At scales greater than $\sim 600 \mathrm{~km}(\sim 0.5 \mathrm{~Hz})$ the spectra show slopes $(1-p) \sim-8 / 3$, which is indicative of Kolmogorov turbulence. From $\sim 600 \mathrm{~km}(\sim 0.5 \mathrm{~Hz})$ to $\sim 100 \mathrm{~km}(\sim 3 \mathrm{~Hz})$ a local flattening is observed, especially for smaller offset distances. At smaller scales a final steepening feature is observed, especially in the egress spectra which sounded small coronal holes. Such a three-component feature is basically consistent with the suggestion by Coles \& Harmon (1989), who compared the results of various observation techniques that provide information on different scales. The present study successfully observed the three components simultaneously in phase spectra for the first time by virtue of the wide spectral coverage.

The transition frequency from the Kolmogorov-like part to the flattened part resembles those seen in the streamer spectra of the Ulysses radio science reported by Pätzold et al. (1996). They reported the break frequencies of $0.06 \mathrm{~Hz}$ and $0.2 \mathrm{~Hz}$ for the coronal hole and streamer spectra, respectively. In our result, however, dependence on the coronal structure is not evident probably because neither dense streamers nor distinct coronal holes were sounded along the ray paths.

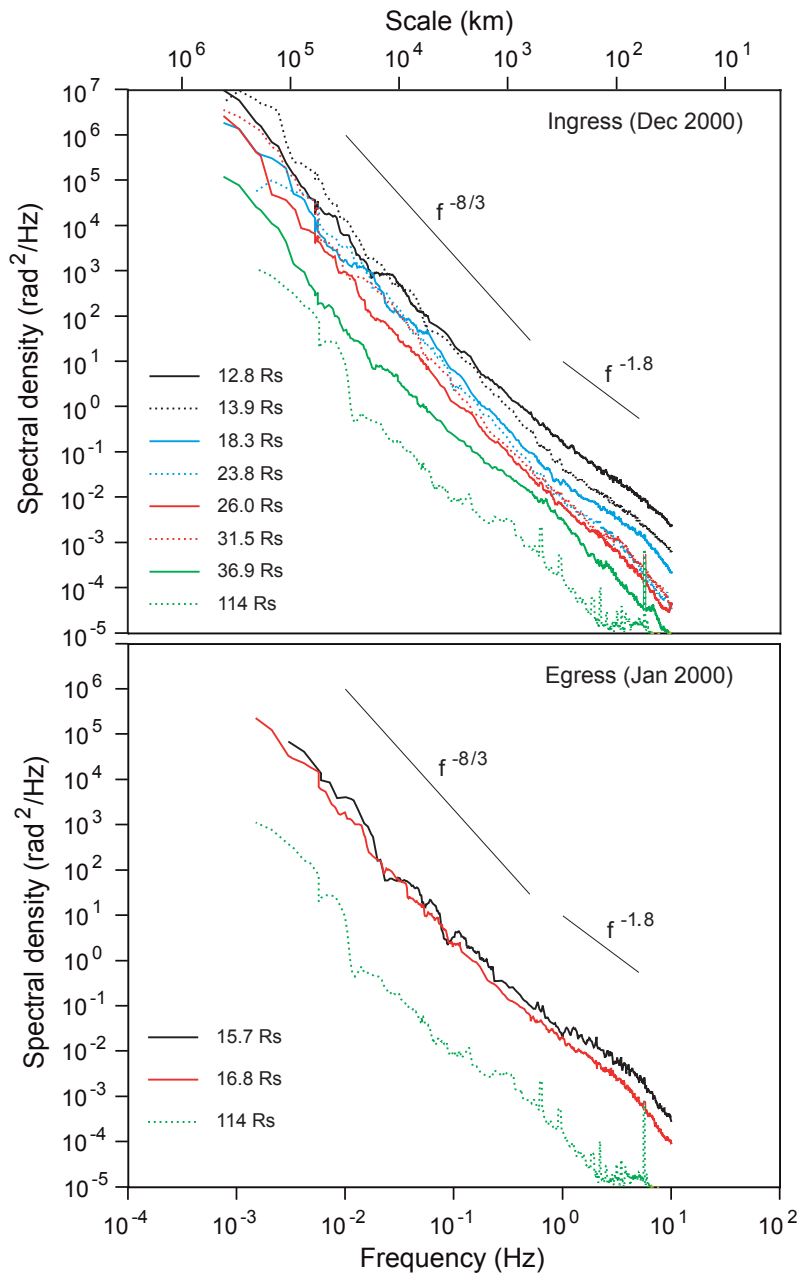

Fig. 4. Phase scintillation spectra obtained in the ingress path (upper panel) and the egress path (lower panel) during the solar conjunction from December of 2000 to January of 2001. Spectral densities are for the $S$-band uplink path, converted from the observed $X$-band spectral densities by multiplying by a factor $(221 / 880)^{2}$. The spectrum observed on June 2, 2000, when the solar offset distance was $114 R_{\mathrm{S}}$, is also plotted for comparison. The spatial scales on the upper axis are based on the solar wind velocity of $300 \mathrm{~km} \mathrm{~s}^{-1}$. The spectral curves have been smoothed for legibility.

\section{Conclusions}

Phase scintillation spectra for solar offset distances of 12.8-36.9 $R_{\mathrm{S}}$ were obtained in two-way mode using the Nozomi spacecraft of Japan. The scintillation level increases as the solar offset distance decreases, which is consistent with previous results (e.g. Woo \& Armstrong 1979). The spectra cannot be represented by a single power law especially for small offset distances (Fig. 4). That is, at low frequencies the spectra show slopes indicative of Kolmogorov, a local flattening is observed from $\sim 0.5 \mathrm{~Hz}$ (scale $\sim 600 \mathrm{~km})$ to $\sim 3 \mathrm{~Hz}(\sim 100 \mathrm{~km})$, and a steepening occurs at higher frequencies. The present study has confirmed the occurrence of local flattening and steepening simultaneously with a single method. We should note, however, that the spectral shape is variable, and the three-component feature is not always evident. The dependence of the spectrum on 
the coronal structure was also suggested, although more experiments will be needed to confirm the tendency.

If the overall Kolmogorov-like slope represents the inertial-range spectrum of magnetohydrodynamic turbulence (Montgomery et al. 1987), the flattening at small scales will reflect enhanced density fluctuations caused by plasma instabilities or waves, and the final steepening at smaller scales will be due to some damping processes (Coles et al. 1991). Grall et al. (1997) found that the microstructure that is responsible for the flattened part is highly field-aligned, and suggested that the enhanced small-scale variance is independent of the background large-scale turbulence and is likely to be due to different physical mechanism. On the other hand, Woo (1996) and Woo \& Habbal (1997) suggested that the break frequency at which the slope changes from Kolmogorov to the flattened one corresponds to the flux tube size in the filamentary structures of the solar wind. In this idea the flattened part represents the density variations across the small-scale irregularities inside flux tubes, while the Kolmogorov part represents the density structures across the filamentary structures co-rotating with the sun. For constraining the small-scale processes in the solar corona, additional phase scintillation observations using spacecrafts in various conditions would be useful. The present study demonstrates that two-way propagation experiments with single downlink frequency still yield valuable information, although the frequency coverage in phase spectra is limited by the cutoff of the transponder. This means that most future deep space missions can perform similar observations even if dualfrequency phase coherent systems are unavailable.

Acknowledgements. We would like to acknowledge E. Mizuno, T. Ichikawa, K. Inoue, T. Takano, M. Yamada, H. Hayakawa, and other members of the Nozomi project team for supporting the observations. The radio science data was acquired by the former graduate students
R. Yoshimura, Y. Koizumi, and Y. Ichikawa and by the staff of the UDSC. This experiment could not have been performed without the assistance of S. Ohashi, M. Sugiura, M. Adachi, F. Fuke and other engineers of NEC TOSHIBA Space Systems, Ltd. We also thank M. Pätzold, A. I. Efimov, and an anonymous reviewer for providing constructive suggestions that greatly improved the paper. The LASCO images we used are the courtesy of the SOHO/LASCO consortium. SOHO is a project of international cooperation between ESA and NASA.

\section{References}

Brueckner, G. E., \& Howard, R. A., Koomen, M. J., et al. 1995, Sol. Phys., 162, 357

Coles, W. A., \& Harmon, J. K. 1989, ApJ, 337, 1023

Coles, W. A., Liu, W., Harmon, J. K., \& Martin, C. L. 1991, J. Geophys. Res., 96, 1745

Grall, R. R., Coles, W. A., Spangler, S. R., Sakurai, T., \& Harmon, J. K. 1997, J. Geophys. Res., 102, 1997

Lipa, B., \& Tyler, L. 1979, Icarus, 39, 192

Mizuno, E., Kawashima, N., Takano, T., \& Rosen, P. A. 1992, IEICE Trans. Commun., E75-B, 665

Montgomery, D., Brown, M. R., \& Matthaeus, W. H. 1987, J. Geophys. Res., 92, 282

Oyama, K.-I., Nabatov, A. S., Savich, N. A., et al. 2001, Adv. Space Res., 27(11), 1847

Pätzold, M., Karl, J., \& Bird, M. K. 1996, A\&A, 316, 449

Woo, R. 1996, Nature, 379, 321

Woo, R., \& Armstrong, J. W. 1979, J. Geophys. Res., 84, 7288

Woo, R., \& Habbal, S. R. 1997, ApJ, 474, L139

Woo, R., Yang, F.-C., Yip, K. W., \& Kendall W. B. 1976a, ApJ, 210, 568

Woo, R., Yang, F.-C., \& Ishimaru, A. 1976b, ApJ, 210, 593

Yakovlev, O. I. 2002, Space Radio Science, ESI Book Series (London: Taylor \& Francis) 